\title{
Bow Shock in Merging Cluster A520: The Edge of the Radio Halo and the Electron-Proton Equilibration Timescale
}

\author{
Qian H. S. Wang ${ }^{1}$ (1) , Simona Giacintucci $^{2}$ (1) , and Maxim Markevitch ${ }^{3,4}$ (C) \\ ${ }^{1}$ Department of Astronomy, University of Maryland, College Park, MD 20742, USA \\ ${ }^{2}$ Naval Research Laboratory, 4555 Overlook Avenue SW, Code 7213, Washington, DC 20375, USA \\ ${ }^{3}$ Astrophysics Science Division, NASA Goddard Space Flight Center, Greenbelt, MD 20771, USA \\ ${ }^{4}$ Joint Space-Science Institute, University of Maryland, College Park, MD 20742, USA \\ Received 2017 June 15; revised 2018 January 29; accepted 2018 February 25; published 2018 April 4
}

\begin{abstract}
We studied the prominent bow shock in the merging galaxy cluster A520 using a deep Chandra X-ray observation and archival VLA radio data. This shock is a useful diagnostic tool, owing to its clear geometry and relatively high Mach number. At the "nose" of the shock, we measure a Mach number of $M=2.4_{-0.2}^{+0.4}$. The shock becomes oblique away from the merger axis, with the Mach number falling to $\simeq 1.6$ around $30^{\circ}$ from the nose. The electron temperature immediately behind the shock nose is consistent with that from the Rankine-Hugoniot adiabat, and is higher (at a 95\% confidence) than expected for adiabatic compression of electrons followed by Coulomb electronproton equilibration, indicating the presence of equilibration mechanisms faster than Coulomb collisions. This is similar to an earlier finding for the Bullet cluster. We also combined four archival VLA data sets to obtain a better image of the cluster's giant radio halo at $1.4 \mathrm{GHz}$. An abrupt edge of the radio halo traces the shock front, and no emission is detected in the pre-shock region. If the radio edge were due only to adiabatic compression of relativistic electrons in pre-shock plasma, we would expect a pre-shock radio emission detectable in this radio data set; however, an interferometric artifact dominates the uncertainty, so we cannot rule this model out. Other interesting features of the radio halo include a peak at the remnant of the cool core, suggesting that the core used to have a radio minihalo, and a peak marking a possible region of high turbulence.
\end{abstract}

Key words: galaxies: clusters: individual (A520) - intergalactic medium - radio continuum: general - X-rays: galaxies: clusters

\section{Introduction}

Galaxy clusters form and grow via mergers of less massive systems. During a merger, shocks and turbulence are generated in the intracluster medium (ICM). They dissipate the kinetic energy of the subclusters (the fraction carried by the ICM) and heat the ICM to the temperature that allows thermal pressure to balance the gravity of the combined massive cluster. Observing the details of this process allows us to gain insight into the complex physics of the magnetized intracluster plasma.

An interesting possibility to observe the process of energy equilibration of the plasma electrons and protons is afforded by shock fronts. In a simple picture, for shocks with low Mach numbers $M$ typical for cluster mergers, the shock passage heats ions dissipatively, while electrons, whose thermal velocity is much higher than that of the shock, are compressed adiabatically. They then equilibrate via Coulomb collisions with protons. If this indeed is how the electron temperature $T_{e}$ behaves in clusters, this would have far-reaching consequences - for example, total mass estimates at large cluster radii, based on the hydrostatic assumption and the electron temperature (e.g., Sarazin 1988), would be biased low because of an underestimate of the average temperature in the low-density cluster outskirts (e.g., Markevitch et al. 1996; Takizawa 1999). This effect has astrophysical implications far beyond galaxy clusters-e.g., certain models of accretion disks rely on this timescale (Rees et al. 1982).

In the X-ray, we directly observe only the electron temperature $T_{e}$, but at an intracluster shock, we can deduce the equilibrium plasma temperature from the directly observable gas density jump, which gives the Mach number.
Luckily, the cluster Mach numbers are low enough for the density jump to be far from its asymptotic value. We can also determine the gas flow velocities on both sides of the shock. We are further lucky that the typical ICM densities and temperatures are such that the product of the Coulomb electron-proton equilibration timescale and the sound speed is of the order of tens of kiloparsecs, which is resolvable by Chandra. This allows us to derive an electron temperature profile across the shock and see if it follows the prediction for collisional equilibration in the narrow zone downstream from the shock. This test has first been applied to the Bullet Cluster (Markevitch 2006, hereafter M06; see also Markevitch \& Vikhlinin 2007, hereafter MV), who obtained a tantalizing conclusion (though only at a 95\% significance) that the equilibration timescale is much shorter than Coulomb. If seen systematically in other cluster shocks, this may suggest the presence of a faster equilibration mechanism in the hot magnetized ICM. While shock fronts are also observed in supernova remnants and even in situ in the solar wind, the electron-proton equilibration timescale can be studied so directly only in cluster shocks, because of the favorable combination of the linear scales and the Mach numbers (e.g., MV).

This test requires a simple, reasonably unambiguous shock geometry and a high-statistics, high-resolution X-ray data set in order to derive a $3 \mathrm{D}$ temperature jump at the shock. After the Bullet cluster result, Russell et al. (2012, hereafter R12) examined two other merger shocks that fit these requirements, those in A2146, but their results were inconclusive because of large uncertainties and the low Mach number of one of the shocks (the difference between shock heating and adiabatic 
Table 1

Details of Archival VLA Observations of A520

\begin{tabular}{|c|c|c|c|c|c|c|c|c|c|}
\hline Project & Configuration & $\begin{array}{c}\text { Frequency } \\
(\mathrm{MHz})\end{array}$ & $\begin{array}{l}\text { Bandwidth } \\
\text { (MHz) }\end{array}$ & Date & $\begin{array}{c}\text { Time } \\
\text { (minutes) }\end{array}$ & $\begin{array}{l}\text { FWHM, PA } \\
\left({ }^{\prime \prime} \times^{\prime \prime},{ }^{\circ}\right)\end{array}$ & $\begin{array}{c}\mathrm{rms} \\
\left(\mu \mathrm{Jy} \mathrm{b}^{-1}\right)\end{array}$ & $\begin{array}{l}\text { Primary } \\
\text { Calibrator }\end{array}$ & $\begin{array}{c}S_{\text {calibrator }} \\
\text { (Jy) }\end{array}$ \\
\hline AF349 & $\mathrm{C}$ & $1364.9 / 1435.1$ & $50 / 50$ & 1998 Dec 8 & 129 & $15.4 \times 14.9,59$ & 25 & $3 \mathrm{C} 48$ & $16.4 / 15.7$ \\
\hline AF349 & $\mathrm{D}$ & $1364.9 / 1664.9^{\mathrm{a}}$ & $50 / 25$ & 1999 Mar 19 & 180 & $50.6 \times 49.4,27$ & 65 & $3 \mathrm{C} 48$ & $16.4 / 14.1$ \\
\hline AC706 & $\mathrm{D}$ & $1364.9 / 1435.1$ & $50 / 50$ & 2004 Aug 13 & 345 & $48.8 \times 46.0,0$ & 50 & $3 C 296$ & $15.4 / 15.0$ \\
\hline
\end{tabular}

Note. Column (1): VLA project identifier; column (2): array configuration; columns (3) and (4): frequency and width of the two intermediate frequency (IF) channels used during the observation; columns (5) and (6): observation date and total time on source; column (7): full width at half-maximum (FWHM) and position angle (PA) of the beam; column (8): image rms noise; columns (9) and (10): primary calibrators and their flux densities set according to the Perley \& Butler (2013) scale.

${ }^{\mathrm{a}}$ We used only the $1364.9 \mathrm{MHz}$ IF channel here.

heating of electrons becomes practically undetectable for $M \lesssim 2$ ). A deep Chandra observation of A520, which we have analyzed in Wang et al. (2016, hereafter W16) for everything else other than the shock front, presents another one of those rare opportunities. We will describe this test in Section 4.2.

Shocks and turbulence generated by the merger would not only heat the intracluster gas, but also accelerate ultrarelativistic particles and amplify magnetic fields that coexist with the thermal plasma. These ultrarelativistic electrons reveal themselves through synchrotron radio emission in the shape of radio halos and relics (e.g., Markevitch et al. 2005; Giacintucci et al. 2008; van Weeren et al. 2010; Feretti et al. 2012; Brunetti $\&$ Jones 2014). While the energy density of these components is a small fraction of the gas thermal pressure, they can substantially alter the physics of the ICM. A520 exhibits a giant radio halo detected by Very Large Array (VLA; Govoni et al. 2001; Vacca et al. 2014), whose distinct brightness edge coincides with the X-ray shock front (Markevitch et al. 2005, hereafter M05), similar to several other clusters with shock fronts (e.g., Giacintucci et al. 2008; Markevitch 2012; Planck Collaboration et al. 2013; Shimwell et al. 2014). The previous analyses of the A520 radio halo used two subsets of VLA data separately, which limited the sensitivity both because of the partial statistical accuracy and the limited coverage of the Fourier space (the $u v$ plane) by the antennas during a typical VLA observation, which may lead to lower reconstructed image fidelity. To take full advantage of the existing radio data, we combine all archival VLA observations in Section 3. We revisit the earlier finding of the coincidence of the radio halo edge and the X-ray shock front. We use the improved radio sensitivity to put an upper limit on the radio emission in the pre-shock region and test one of the possible mechanisms for the origin of the radio edge considered in M05-adiabatic compression of pre-existing relativistic electrons. There are other illuminating coincidences between the radio and X-ray structure of the cluster that we discuss in Section 4.3.

We assume a flat cosmology with $H_{0}=70 \mathrm{~km} \mathrm{~s}^{-1} \mathrm{Mpc}^{-1}$ and $\Omega_{m}=0.3$, in which $1^{\prime \prime}$ is $3.34 \mathrm{kpc}$ at the cluster's redshift of 0.203 . Uncertainties are quoted at $90 \%$ confidence unless otherwise stated.

\section{X-Ray Data Analysis}

The Chandra data reduction is described in W16, where we discussed all the A520 features seen in this data set other than the shock front. In summary, we use $447 \mathrm{ks}$ of Chandra observations of A520 performed in 2007-2008 (ObsIDs 9424, 9425, 9426, 9430). Three earlier observations (ObsIDs 528,
$7703,4125)$ were not used because they would not meaningfully improve our results while adding complexity to the analysis. Spectral analysis was performed using XSPEC (v12.9.0). Temperatures were obtained by fitting an absorbed APEC model, while accounting for the additional background component as determined in W16. The redshift was fixed at $z=0.203$, while metal abundance (relative to Anders \& Grevesse 1989) and Galactic absorption were fixed at the bestfit cluster average values of 0.21 and $N_{H}=6.3 \times 10^{20} \mathrm{~cm}^{-2}$, respectively, obtained from a fit to the spectrum from an $r=3^{\prime}$ circle centered on the X-ray centroid.

\section{Radio Data Analysis}

We reanalyzed the archival VLA data at $1.4 \mathrm{GHz}$ from project AF349 (the data used in Govoni et al. 2001) and projects AC776 and AC706 (the data used in Vacca et al. 2014), which observed A520 in C and D array configurations. Table 1 gives technical details of these observations.

We calibrated and reduced the data sets separately using the Astronomical Image Processing System (AIPS). We followed the standard procedure, with amplitude and phase calibration carried out after accurate editing of the raw data on both the primary and secondary calibration sources. The flux density scale was set using the amplitude calibrators listed in Table 1 and the Perley \& Butler (2013) coefficients in AIPS SETJY task. The accuracy of the flux density scale is estimated to be within $3 \%$. Phase-only self-calibration was applied to each data set to reduce the effects of residual phase errors. Final images were made using the multi-scale CLEAN algorithm implemented in AIPS IMAGR task, which results in better imaging of the extended sources compared to the traditional single-resolution CLEAN (e.g., Clarke \& Ensslin 2006). After self-calibration, we combined the $\mathrm{C}$ and $\mathrm{D}$ data into a single data set. For the AF349 D-configuration observation, we used only the 1364.9 MHz IF channel that matches the frequency and width of the first IFs of all other data sets. Finally, a further cycle of phase calibration was applied to the combined data set to improve the image quality. We reached an rms sensitivity level of $20 \mu \mathrm{Jy}_{\text {beam }}{ }^{-1}$ in the final combined image, with a restoring beam of $19^{\prime \prime}$.

Good sampling of short baselines (i.e., close antenna pairs) in the $u v$ plane is crucial for correct determination of the flux density, size, and structure of a low-surface-brightness source like the radio halo in A520. The inner portion of the $u v$ plane of our final combined data set is shown in Figure 1. Only visibilities corresponding to baselines shorter than $1.5 \mathrm{k} \lambda$ are plotted. The very good sampling of short spacings in this plot 


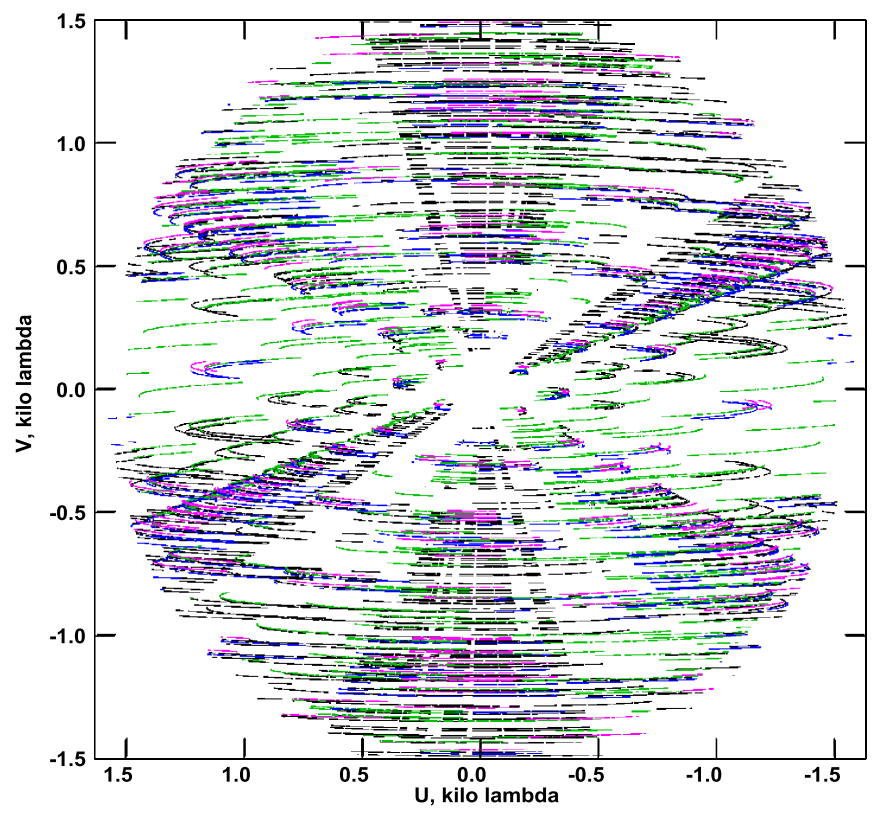

Figure 1. Coverage of the $u v$ plane of spatial frequencies by the four combined VLA data sets (shown by different colors). Fuller coverage results in a reconstructed image with higher fidelity for the extended features. The data sets are complementary, especially at smaller wavenumbers near the center of the plot (corresponding to larger angular scales).

ensures high-fidelity imaging of the radio halo whose angular size of about $5^{\prime}$ (diameter) is sampled by visibilities shorter than $0.8 \mathrm{k} \lambda$. Nominally, the largest structure detectable by this data set should be about $3 \mathrm{Mpc}$; we will return to this in a more quantitative way below.

To image only the extended and compact radio sources unrelated to the giant halo, we produced images using only the baselines longer than $0.5 \mathrm{k} \lambda$ and longer than $1 \mathrm{k} \lambda$, respectively. We identified 16 such sources with peak flux densities exceeding a $3 \sigma$ level of $0.06 \mathrm{mJy}^{\text {beam }}{ }^{-1}$ (for a $19^{\prime \prime}$ restoring beam) within $r \sim 1 \mathrm{Mpc}$ from the cluster X-ray centroid. These include three extended radio galaxies (two with the narrowangle tail morphology and one a double-lobed source), one marginally resolved object (a possible "dying" radio galaxy, as discussed by Vacca et al. 2014), and 12 unresolved sources. We then subtracted the CLEAN components associated with these compact sources (for a total flux density of $75 \mathrm{mJy}$ ) from the $u v$ data and used the resulting data set to obtain images of only the diffuse radio halo at multiple resolutions using the multi-scale CLEAN. Images restored with a $22^{\prime \prime}$ circular beam before and after the removal of the sources unrelated to the halo are shown in Figure 2. The image with sources removed (panel (a)) has an rms noise level of $22 \mu \mathrm{Jy}_{\text {beam }}{ }^{-1}$. The halo flux density, measured within the $1 \sigma$ isocontour, is $20.2 \mathrm{mJy}$ with an error of $7.2 \%$, computed following Cassano et al. (2013), i.e., including the flux calibration uncertainty (3\%), the noise in the integration area, and the error due to the subtraction of the discrete radio sources in the halo region. Our flux measurement is in agreement with the flux density of $19.8 \pm$ 1.4 used by Cassano et al. (2013) to calculate the radio halo luminosity at $1.4 \mathrm{GHz}$ and measured on an image obtained from the AF349 observations. A slightly lower flux of $16.7 \pm$ $0.6 \mathrm{mJy}$ is measured by Vacca et al. (2014) by masking the radio galaxies (rather than subtracting them as we did).
Our high-quality image of the radio halo reveals a prominent edge and significant brightness structure on small angular scales. We will compare these fine features with our X-ray data in Sections 4.3 and 4.4. As we will see, the data set still exhibits some interferometric artifacts with the scale and amplitude that are significant for us; we will address this in Section 4.4.1.

\section{Discussion}

\subsection{Bow Shock}

A classic bow shock would exhibit the highest Mach number $M$ (and the highest gas density and temperature jumps) at the "nose," and a decreasing $M$ as the angle from the axis of symmetry of the shock increases and the shock becomes oblique. For the electron-proton equilibration test that we want to perform, we need as high a Mach number as possible to maximize the difference in electron temperature between the two possibilities, and thus want to study as narrow a sector at the "nose" as possible. The deep A520 X-ray observation provides sufficient statistics to analyze the bow shock in several sectors, divided based on the brightness contrast across the front (Figure 3(a)). We exclude a narrow segment of the front between sectors $\mathrm{S}$ and N1 immediately in front of the bright cool structure because of the small-scale irregularities, possibly caused by the dark matter mass peak located there (see Figure 6 in W16), that would be difficult to model. A small region that includes those structures is also excluded from sector $\mathrm{N} 1$ as shown in Figure 3(a).

In each sector, we fit the $0.8-4.0 \mathrm{keV}$ surface brightness profile with a density model that consists of two power-law radial profiles (with different slopes) on either side of the shock and an abrupt jump at the shock, whose position is a free parameter. This 3D model is projected onto the sky under the assumption that the curvature along the line of sight (los) is the same as that of the brightness edge in the plane of the sky (which is further discussed in Section 4.2.1) and compared to the brightness profiles extracted in the respective sector. For these observations, we can use the $0.8-4.0 \mathrm{keV}$ count rate as a direct proxy for the los-integrated $n_{e}^{2}$, because the combination of the spectral model parameters $\left(N_{H}\right.$, abundance, gas temperatures) and the Chandra response in this energy band conspire to make the dependence of the $\mathrm{X}$-ray flux on temperature negligible $(<1 \%$ for the interesting range of temperatures, based on examining how the predicted flux responds to varying the plasma temperature of the model in XSPEC). The Mach number, $M$, of the shock front relative to the upstream flow is derived from the density jump $x$ using the Rankine-Hugoniot jump conditions (Landau \& Lifshitz 1959):

$$
M=\left(\frac{2 x}{(\gamma+1)-(\gamma-1) x}\right)^{1 / 2}
$$

where we use the adiabatic index $\gamma=5 / 3$ for monatomic gas. For typical, low Mach numbers found in clusters, $x$ is far from the asymptotic value for strong shocks (4 for $\gamma=5 / 3$ ) and thus allows an accurate determination of $M$. Fit results for the sectors are shown in Table 2. The highest Mach numbers, $M=2.5_{-0.4}^{+0.5}$ and $M=2.4_{-0.3}^{+0.6}$, are seen in sectors N1 and N2 at the "nose" of the shock, respectively, and decrease to $M<2$ on either side, where the shock becomes oblique. The shock "nose" 

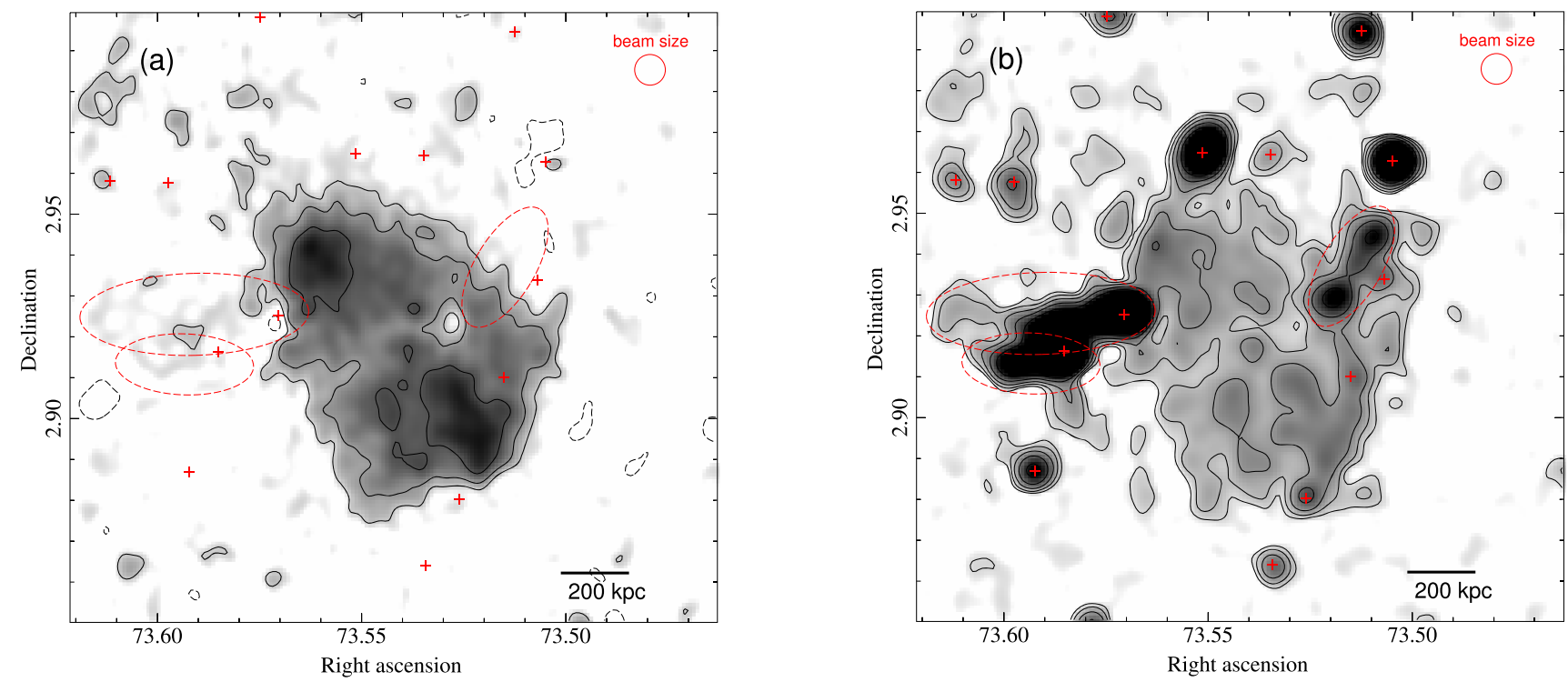

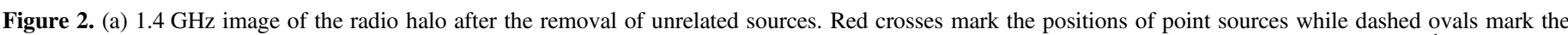

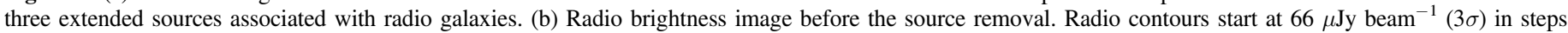
of $\times 2$ (dashed contours are negative). The beam size is the same in both images. $200 \mathrm{kpc}$ is $1^{\prime}$.

direction is in agreement with the NE-SW merger axis. The "nose" sectors have higher values compared to $M=2.1_{-0.3}^{+0.4}$ reported in M05 because the latter included the adjacent sectors with lower density jumps.

Although the $M$ decline toward the wings of the front is expected, it has only been reported previously for the main shock of the Bullet cluster (MV), because such a study requires good statistics. Care should therefore be taken when using a wide sector to analyze bow shocks, as the peak density jump will probably be underestimated.

\subsection{Electron-Ion Equilibration Timescale}

In the collisional plasma picture, a shock front with relatively low Mach numbers-such as those occurring in cluster mergers -would heat protons and heavier ions dissipatively, while electrons, whose thermal velocity is much higher than the velocity of such shocks, are compressed adiabatically to a temperature lower than that of ions. Protons and electrons subsequently equilibrate on a Coulomb collision timescale (e.g., Spitzer 1962; Zeldovich \& Raizer 1966):

$$
\tau_{\mathrm{ep}}=2 \times 10^{8} \text { year }\left(\frac{n_{e}}{10^{-3} \mathrm{~cm}^{-3}}\right)^{-1}\left(\frac{T_{e}}{10^{8} \mathrm{~K}}\right)^{3 / 2} .
$$

We can measure the electron temperature directly by modeling the X-ray spectrum, but cannot measure the proton temperature. However, the equilibrium post-shock temperature $\overline{T_{2}}$ (the one that protons and electrons achieve asymptotically) can be derived from the pre-shock temperature and the compression factor $x$ (or, equivalently, the Mach number) using the shock jump conditions:

$$
\frac{\overline{T_{2}}}{T_{1}}=\frac{(\gamma+1) x-\gamma+1}{x(\gamma+1)-x^{2}(\gamma-1)} .
$$

Indices 1 and 2 correspond to pre-shock and post-shock quantities, respectively. The time dependence of the electron temperature $T_{e}$ increasing asymptotically from the adiabatic value to the equilibrium value under Coulomb collisions was given by, e.g., Fox \& Loeb (1997), Wong \& Sarazin (2009), and Sarazin et al. (2016). As the local $T_{e}$ increases, the post-shock gas flows away from the shock front, and the $T_{e}$ time dependence gets encoded in the spatial temperature profile, which can be measured by Chandra. The closing piece of the experimental setup is the velocity of the post-shock gas relative to the front, which is given by the shock mass conservation condition:

$$
v_{2}=M c_{s 1} / x,
$$

where $c_{s 1}$ is the pre-shock sounds speed, determined from the $\mathrm{X}$-ray measured pre-shock temperature (electrons and ions in the pre-shock region can reasonably be assumed in equilibrium).

The Mach number should be sufficiently high to distinguish between shock heating and adiabatic compression. For $M=2.4$, there is a measurable difference between the two, but they become practically indistinguishable for $M \lesssim 2$. In sectors N1 and N2, the shock is strongest, and their Mach numbers are statistically the same (Table 2), thus we will combine them (see the $\mathrm{N} 1+\mathrm{N} 2$ entry) and use the combined profile for the above test. We will use the other sectors, which should be insensitive to the possible temperature nonequilibrium, for consistency checks.

We construct two model $T_{e}$ profiles for each sector, one for adiabatic compression at the shock and subsequent Coulomb equilibration, and the other for instant equilibration, and compare them with the observed temperature profile. The electron density is derived from the emission measure using the normalization of the APEC model in XSPEC, $\int n_{e} n_{H} d V=$ $10^{14} \times$ norm $\times 4 \pi\left[D_{A}(1+z)\right]^{2}$. In sector $\mathrm{N} 1+\mathrm{N} 2$, we find $n_{e 1}=(4.04 \pm 0.15) \times 10^{-4} \mathrm{~cm}^{-3}$ immediately in front of the shock, and $n_{e 2}=(1.07 \pm 0.11) \times 10^{-3} \mathrm{~cm}^{-3}$ behind the shock (the latter includes a $10 \%$ uncertainty of the density jump). Reasonable deviation of the shock surface from spherical does not affect our results-this is discussed in Section 4.2.1. 

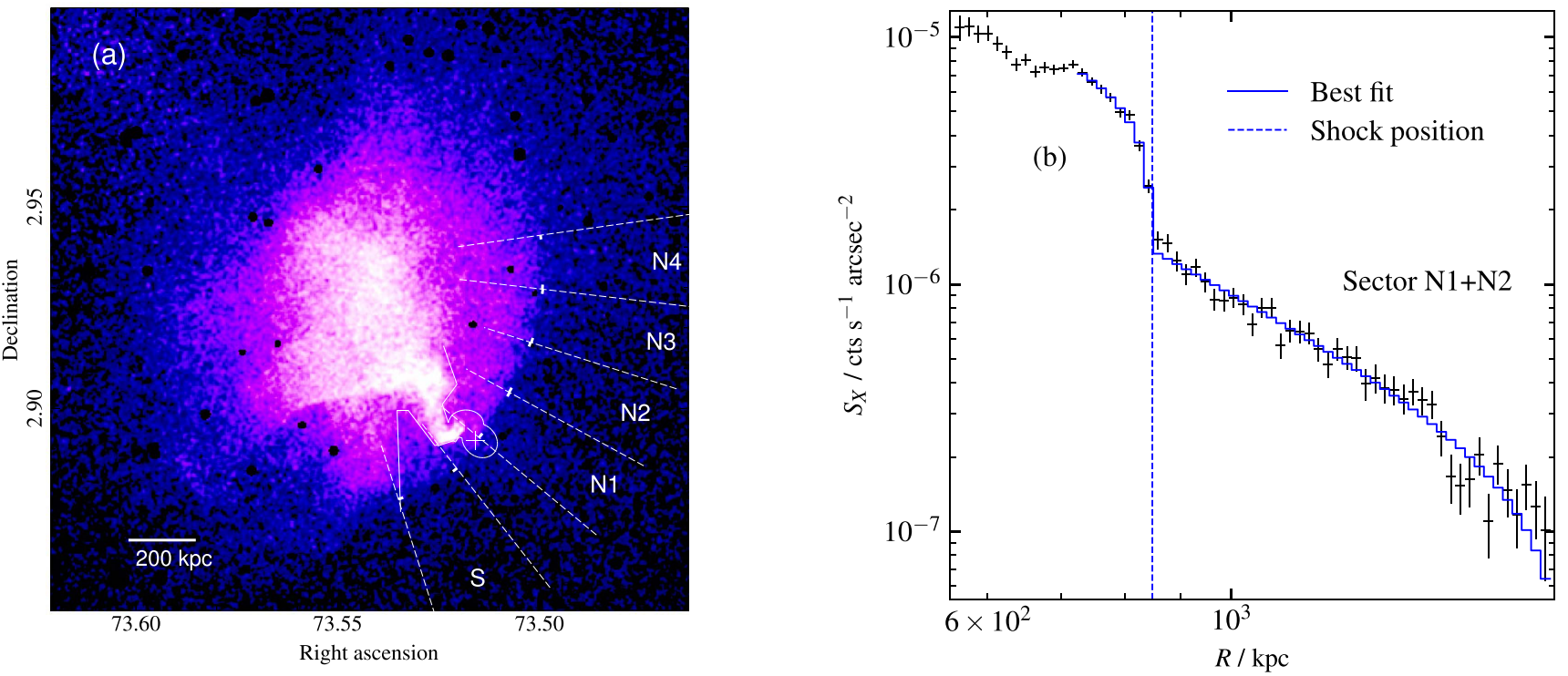

Figure 3. (a) 0.8-4 keV Chandra image, smoothed by $2^{\prime \prime}$ Gaussian (holes are masked point sources). The white dashed lines mark the sectors used for X-ray shock profile fitting, with tick marks indicating the best-fit shock position in each sector. The white cross indicates the position of the BCG next to the cold front. The white solid outline indicates masked regions for the X-ray brightness profile and spectral extraction, covering the cold front close to the shock surface. (b) X-ray brightness profile in the combined sector $\mathrm{N} 1+\mathrm{N} 2$ and best-fit model. $200 \mathrm{kpc}$ is $1^{\prime}$.

Table 2

Details of X-Ray Modeling of Shock Sectors

\begin{tabular}{lccccc}
\hline \hline Sector & $\rho$ Jump & $M$ & Inner Slope & Outer Slope & $\chi^{2} / \nu$ \\
\hline S & $2.0_{-0.3}^{+0.2}$ & $1.7_{-0.2}^{+0.2}$ & $-0.8_{-0.6}^{+0.4}$ & $-1.8_{-0.2}^{+0.3}$ & $59.5 / 43$ \\
N1 & $2.7_{-0.3}^{+0.3}$ & $2.5_{-0.4}^{+0.5}$ & $-0.3_{-0.7}^{+0.7}$ & $-1.7_{-0.2}^{+0.3}$ & $35.6 / 27$ \\
N2 & $2.6_{-0.2}^{+0.4}$ & $2.4_{-0.3}^{+0.6}$ & $-0.2_{-0.6}^{+0.7}$ & $-1.7_{-0.3}^{+0.2}$ & $34.3 / 27$ \\
N3 & $2.2_{-0.2}^{+0.3}$ & $1.9_{-0.2}^{+0.3}$ & $-1.1_{-0.8}^{+0.6}$ & $-1.7_{-0.2}^{+0.2}$ & $44.2 / 27$ \\
N4 & $1.9_{-0.2}^{+0.2}$ & $1.6_{-0.1}^{+0.2}$ & $-0.4_{-0.3}^{+0.3}$ & $-1.5_{-0.2}^{+0.1}$ & $68.5 / 39$ \\
N1+N2 & $2.7_{-0.3}^{+0.2}$ & $2.4_{-0.2}^{+0.4}$ & $-0.5_{-0.7}^{+0.8}$ & $-1.6_{-0.2}^{+0.1}$ & $24.7 / 30$
\end{tabular}

Note. Column (1): shock sector as shown in Figure 3(a); column (2): density jump at shock; column (3): shock Mach number; column (4): density profile inner power-law index; column (5): density profile outer power-law index; column (6): chi-square and degree of freedom. Errors are $90 \%$ with all other parameters free.

The pre-shock temperature profile for $\mathrm{N} 1+\mathrm{N} 2$ is consistent with being constant out to at least $800 \mathrm{kpc}$ from the shock (Figure 4). We thus decided to use the best-fit temperature in a radial bin between 10 and $400 \mathrm{kpc}$ from the shock (where we excluded the immediate vicinity of the shock to avoid any irregularities of the front), $T=4.70_{-0.72}^{+0.82} \mathrm{keV}$, as the pre-shock value. This temperature, the best-fit compression factor, and Equation (4) give the post-shock gas velocity of $1030_{-80}^{+90} \mathrm{~km} \mathrm{~s}^{-1}$ relative to the shock front. During the collision equilibration timescale $\tau_{\mathrm{ep}} \simeq 0.2 \mathrm{Gyr}$, the post-shock gas travels $\sim 200 \mathrm{kpc}$ or 65", which is well resolved by Chandra.

We will compare the deprojected and projected temperature profiles for $\mathrm{N} 1+\mathrm{N} 2$ with the instant-equilibration and adiabatic compression models in Figure 4 and Figure 5(a). To construct the adiabatic compression model, we calculate the time dependence of the local post-shock $T_{e}$ using the measured shock parameters following Sarazin et al. (2016). For the instantequilibration model, we assume the electron temperature jumping to $\overline{T_{2}}$ right at the shock. These models with their uncertainties, which include statistical uncertainties of the preshock temperature and the density jump, are shown in Figure 4.

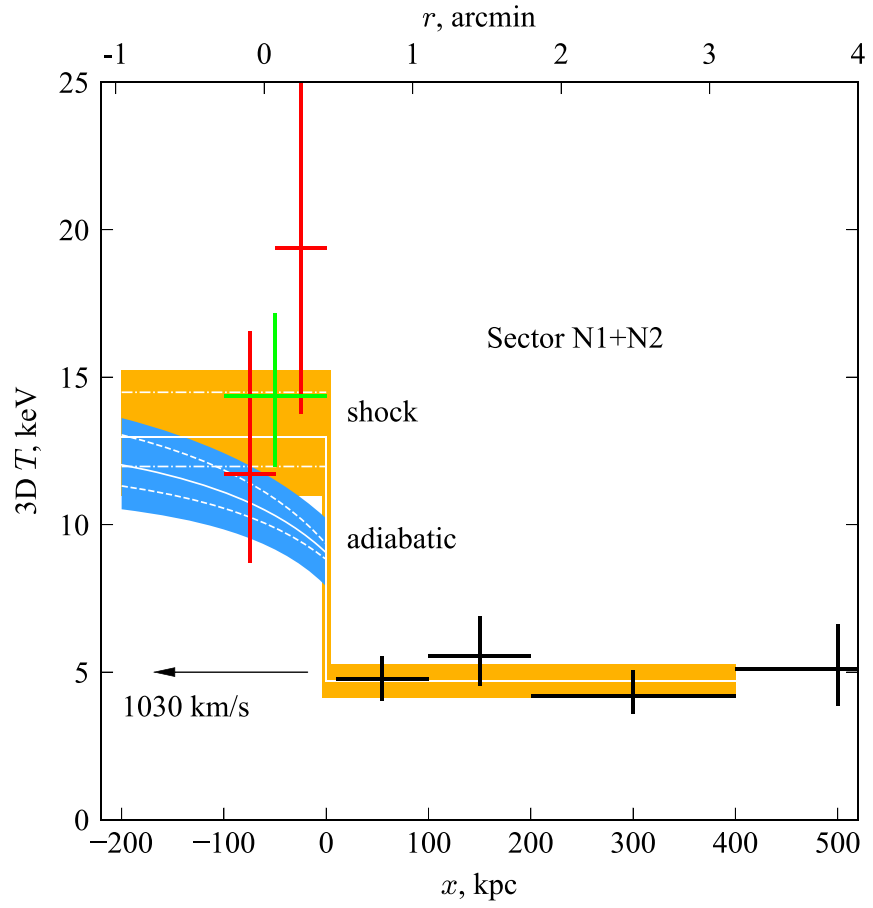

Figure 4. Deprojected post-shock temperatures compared with model profiles in sectors N1 and N2 combined (Figure 3(a)). The yellow band is the instantequilibration model, while the blue band is adiabatic compression followed by Coulomb equilibration. The bandwidth indicates $1 \sigma$ error bounds. In the preshock region, this equals the error in the pre-shock temperature measurement, while in the post-shock region this further includes the density jump parameter uncertainty, which has a smaller effect. The white dashed and dotted-dashed lines bounding the post-shock model profiles indicate the effect of geometric uncertainty ( $\pm 10 \%$ change in the los extent). The $x$-axis denotes distance from the shock position. Different colors of post-shock crosses correspond to bins of different widths (red is $50 \mathrm{kpc}$ and green is $100 \mathrm{kpc}$ ). $x$-error bars denote the radii in which temperature was measured. $y$-errors are $1 \sigma$.

The 3D temperature model profile is projected onto the sky using the best-fit density model and the spectroscopic-like temperature weighting $w=n^{2} T^{-3 / 4}$, following Mazzotta et al. (2004). The 

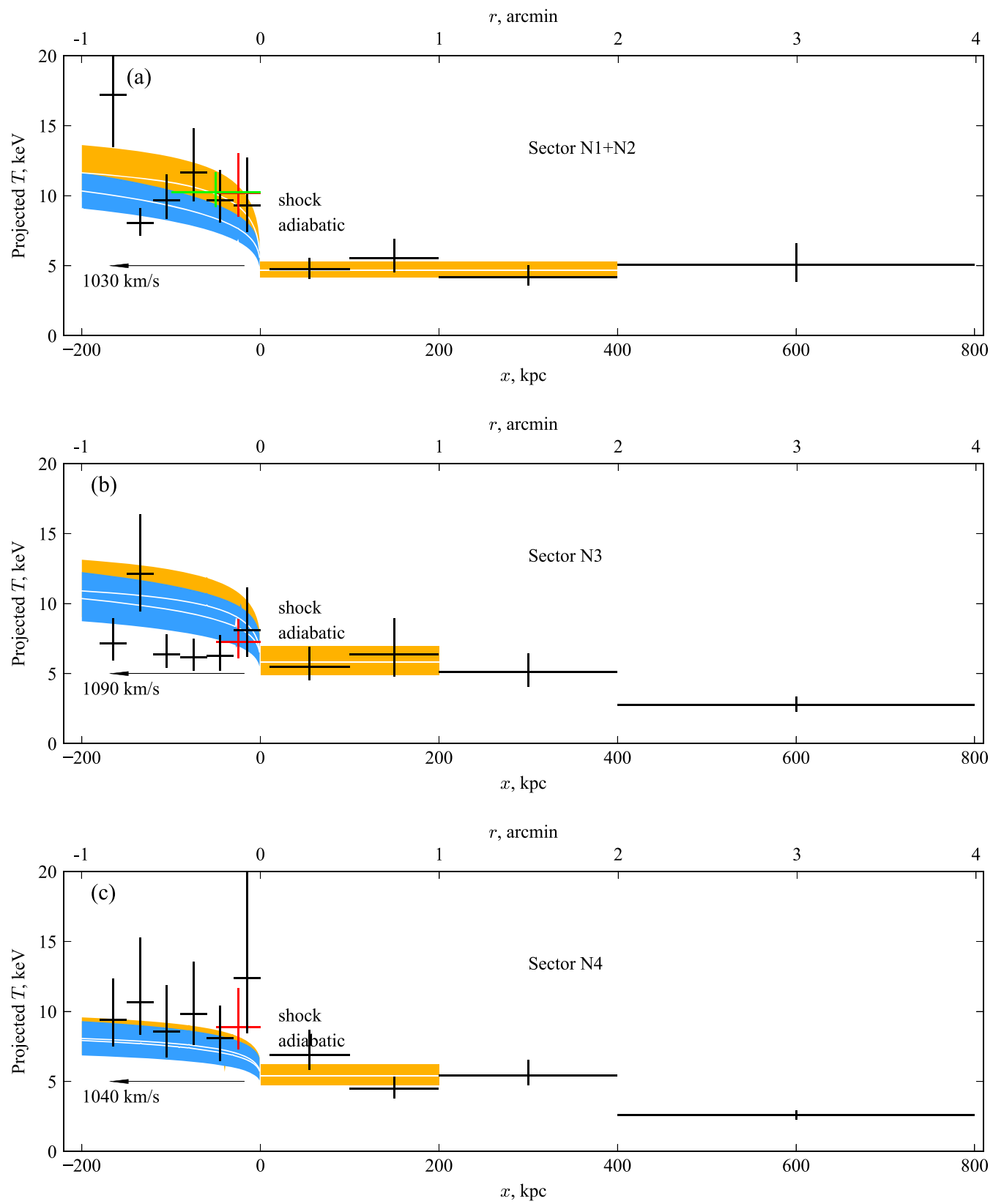

Figure 5. Projected temperatures compared with model profiles for segments of the shock surface in various sectors labeled in Figure 3(a). (a) Projected spectroscopiclike temperature in sector N1+N2 (using $n^{2} T^{-3 / 4}$ weighting; following Mazzotta et al. 2004). (b) Projected temperature in sector N3. (c) Projected temperature in sector N4. The $x$-axis denotes distance from the shock position. Different colors of post-shock crosses correspond to bins of different widths. $x$-error bars denote the radii in which temperature was measured. $y$-errors are $1 \sigma$. The bands are $1 \sigma$ error bounds.

projected model profiles are shown in Figure 5 for sector $\mathrm{N} 1+$ $\mathrm{N} 2$ as well as N3 and N4.

We also deproject the measured temperatures in bins of several sizes $(30,50,100 \mathrm{kpc})$ immediately after the shock by estimating the contributions of the outer $3 \mathrm{D}$ shells into the spectrum from the respective post-shock region and adding the properly normalized spectral component in the XSPEC fit to represent the projected gas. Using the projected profile and using the deprojected profile are equivalent-the difference between the two models is greater for the $3 \mathrm{D}$ profiles, but so is the uncertainty of the deprojected measured temperature.

For the $\mathrm{N} 1+\mathrm{N} 2$ sector, in the $50 \mathrm{kpc}$ bin behind the shock, we measure the projected temperature of $T=10.2_{-2.4}^{+5.3} \mathrm{keV}$, while the deprojected temperature using the best-fit $3 \mathrm{D}$ density model is
$T=19.4_{-8.4} \mathrm{keV}$ (unconstrained on the high side because of Chandra's poor sensitivity to such high temperatures). The first post-shock bin is the most useful, because it has the greatest model difference. For illustration purposes, we also obtained a deprojected temperature for the second $50 \mathrm{kpc}$ shell, with the first $50 \mathrm{kpc}$ post-shock shell fixed at the model instant-equilibration temperature, in effect deprojecting the instant-equilibration model (this is done to regularize the deprojection procedure, as the errors of the neighboring bins are anti-correlated). The procedure was repeated for three narrower $30 \mathrm{kpc}$ post-shock bins, and for one wider $100 \mathrm{kpc}$ bin.

The deprojected $T_{e}$ in the first 30,50 , and $100 \mathrm{kpc}$ postshock bins are all above the adiabatic model and consistent with the instant-equilibration model. (Of course, these 
measurements are not statistically independent.) The adiabatic model is below the measured deprojected value at $95 \%$ significance in a single parameter test for the $50 \mathrm{kpc}$ and $100 \mathrm{kpc}$ bins, and around $90 \%$ for the $30 \mathrm{kpc}$ bin. Similarly, the projected spectroscopic-like temperatures are higher than the adiabatic compression model at 95\% significance for the $50 \mathrm{kpc}$ and $100 \mathrm{kpc}$ bins.

In the 30 and $50 \mathrm{kpc}$ bins further from the shock, the temperatures remain consistent with the instant-equilibration model. In Figure 5(a), we show the $30 \mathrm{kpc}$ bins for the projected profile up to about $200 \mathrm{kpc}$ behind the shock to give a broader overview; however, cool core fragments and unrelated cluster structure can be seen within $200 \mathrm{kpc}$ behind the shock (Figure 3(a)), which can affect the projected temperatures and create the apparent large scatter.

For a consistency check, we also obtained the projected temperature profiles in sectors $\mathrm{N} 3\left(M=1.9_{-0.2}^{+0.3}\right)$ and N4 $\left(M=1.6_{-0.1}^{+0.2}\right)$, where the Mach numbers are insufficiently high to distinguish the two models (Figures 5(b), (c)). In these sectors, the pre-shock temperature shows a slow decrease with radius, so we derived the best-fit pre-shock temperatures in a narrower $10-200 \mathrm{kpc}$ bin. They are consistent with the preshock temperature for $\mathrm{N} 1+\mathrm{N} 2$. In both sectors, the temperature increase immediately behind the shock is consistent with both models. In N3, the presence of a cool blob of gas causes measurements from about $50 \mathrm{kpc}$ behind the shock to fall down; this blob has been seen in the temperature map (Figure 2 in W16). In N4, measurements appear systematically above the models (although not significantly). This may be caused by our underestimating of the immediate pre-shock temperature, as the deviation at the first pre-shock bin suggests. We conclude that sectors N3 and N4 behave consistently with the expectation for the lower Mach numbers observed in these sectors.

\subsubsection{Geometrical Systematic Uncertainty}

In the above experiment, we relied on the assumption that the shock surface has the same curvature along the los as in the plane of the sky. This is a reasonable assumption for this merger with a relatively clear geometry, for which the apparent shock direction is generally well aligned with the merger axis evident from both the X-ray and lensing maps, and the shock front center of curvature in the image approximately coincides with the large-scale cluster centroid. Nevertheless, we should determine how the uncertainty of this assumption affects the results. If the surface has a different curvature along the los, we would derive the incorrect density jump and Mach number. The deprojected post-shock temperature would also be affected, but because of the relatively high brightness contrast at the shock (i.e., a relatively low projected contribution), this is a secondary effect.

To evaluate the effect, we varied the radius of curvature of the shock surface along the los, while keeping the pre-shock gas model unchanged (spherically symmetric). For simplicity we used a spheroid geometry for the shock surface, keeping its axes in the plane of the sky to be the same as the shock radius $r_{\text {jump }}$, while linearly stretching its los axis. Note that with this geometry, the extent $l$ of the shock surface in the los direction scales with the los radius of curvature $R$ not linearly but as $l \propto \sqrt{R}$.

For a $20 \%$ change in $R$, the best-fit density jump changed by $5 \%$ - the difference coming from the change in post-shock density, while the pre-shock density stays the same. This is smaller in magnitude than the $\sim 10 \%$ fitting error on this parameter, so for a moderate amount of shock-surface variation, the geometry does not significantly affect our results (see Figure 4). For this uncertainty to become dominant, the shock surface should be very asymmetric, e.g., a factor of 1.7 difference in $R$ corresponds to a $15 \%$ change to the density jump.

\subsubsection{Comparison with Other Shocks}

While such a degree of asymmetry seems unlikely for the relatively symmetric merger in A520, there is no way of knowing this for sure for each individual shock. One way to assess the probability of the shock front asymmetries and how well the true Mach number is recovered from the X-ray density profiles is to study shocks in cosmological simulations. On the observational side, measurements for a sample of relatively strong $(M \gtrsim 2.5)$ shocks is needed for a robust conclusion on the electron-proton equilibration timescale. Our A520 result adds a data point to two other previously published measurements-the Bullet cluster with $M \approx 3$ (M06, MV) and the stronger of the two shocks in A2146, one with $M=2.3$ (R12). The Bullet cluster showed, at a similar 95\% confidence, a similar preference for fast electron-proton equilibration. The A2146 shock showed preference for the Coulomb equilibration at a similar $\sim 2 \sigma$ significance (considering, as we do, only the temperature bin immediately after the shock)—although the instant-equilibration and Coulomb models themselves were only $1 \sigma$ apart due to a low $M$ and a large uncertainty for the pre-shock temperature. The physics of the intracluster plasma in different clusters should be similar, so we should get the same answer from all of the experiments. The mild contradiction between the Bullet and A520 on one side and A2146 on the other may be a reflection of the above geometrical uncertainty. We do note that the bow shock in A2146 used in R12 exhibits a flat shape at its "nose," with the shock center of curvature far from the cluster centroid (see Figure 8 in R12), which diminishes our confidence in the above los/image plane symmetry assumption. It is interesting that their second, weaker shock exhibits a $T_{e}$ jump that is higher than the prediction of both models (at a similar significance), which may be further illustration of the geometric uncertainty. An additional apparent difference between our (along with M06 and Sarazin et al. 2016) and R12 analyses is the three times longer Coulomb equilibration timescale used in R12 (cf. their Equation (2) and our Equation (2)), although this would not reconcile the results.

Sarazin et al. (2016) performed a similar test on an $M=2.5$ shock front at the position of the western radio relic in A3667. Their derived post-shock electron temperature goes below even the adiabatic model, which would appear to indicate a problem with this method. However, that shock is located $2 \mathrm{Mpc}$ away from the cluster center, where the cluster emission is very faint and a projection of any unrelated X-ray structure on the los may have a significant effect. For example, if a faint, cool group were projected onto the post-shock region, it would result in both an overestimate of the gas density jump and an underestimate of the temperature jump-effect of the right sign to explain their result. For A520, as well as the Bullet and A2146, projection of unrelated objects is much less of a problem because the shocks are located in much brighter cluster regions. 

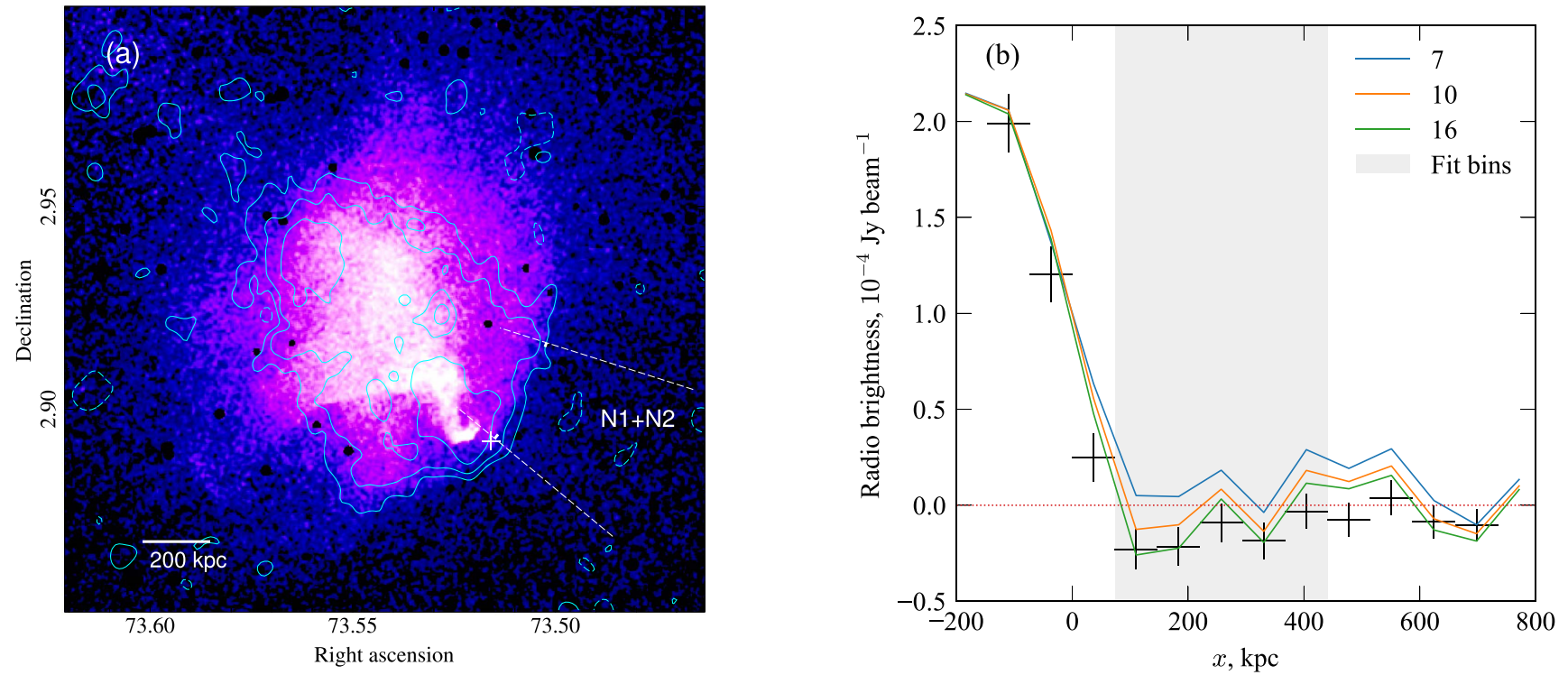

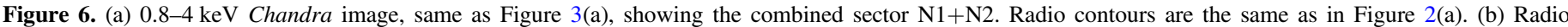

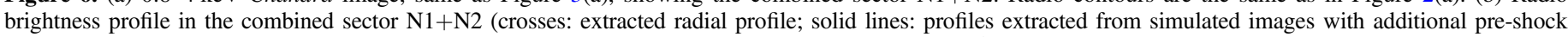

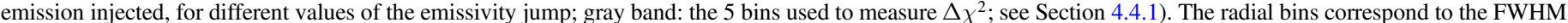
beam size. $x$ is relative to the X-ray best-fit shock position. $200 \mathrm{kpc}$ is $1^{\prime}$.

Thus, our conclusion is score 2:1 in favor of quick electronproton equilibration in the intracluster plasma, but more strong shocks need to be studied to reduce the systematic uncertainties.

\subsection{Radio Halo Features}

There are interesting coincidences between the radio halo and X-ray features in A520 (Figure 6(a)). There are bright radio spots at the positions of the cool "foot" and "knee" that we discussed in W16. The radio emission here may be related to a radio minihalo that had inhabited the cool core before its disruption, which gave rise to these cool X-ray clumps, as minihalos are observed in almost all massive cool cores (Giacintucci et al. 2017). The radio enhancements there may also be caused by reacceleration of relativistic particles by local turbulence in the wake of the disrupted cool core (see Brunetti \& Jones 2014 for review of possible acceleration mechanisms in clusters). Another prominent, broad brightness peak in the $\mathrm{NE}$ half of the radio halo is located at one of the hottest regions of the cluster, but it does not have any obvious corresponding $\mathrm{X}$-ray brightness structures. This can be the site of vigorous merger-induced turbulence, which would produce relativistic electrons via reacceleration. Future spatially resolved X-ray calorimeters, such as $X A R M$, will be able to detect this turbulent region.

\subsection{Origin of the Radio Edge}

As discussed in M05, the X-ray bow shock in A520 traces a sharp edge of the radio halo, and we see it clearly in Figure 6. Mechanisms of producing ultrarelativistic electrons responsible for the post-shock radio synchrotron emission include firstorder Fermi acceleration, which can use thermal electrons as its seeds or re-accelerates "fossil" relativistic electrons (e.g., Blandford \& Eichler 1987) that existed prior to shock passage but whose radio brightness is below the detection limit. Another possible mechanism is adiabatic compression of such fossil electrons and the compression of the magnetic field (since cosmic rays and magnetic fields are frozen into the thermal gas that is being compressed by the shock). Both the adiabatic compression and the reacceleration should be present, but the reacceleration boost for aged, steep-spectrum electrons depends on the the Lorentz factor $\gamma_{\max }$ of the fossil electrons (see M05 for discussion of the resulting spectrum and normalization), so either effect may dominate. In either of these two scenarios, the fossil relativistic electrons in the preshock region should produce radio emission at a certain low brightness level that can be related to that of the post-shock emission. As derived in M05 in the compression-only scenario, for a power-law fossil electron energy spectrum with index $\delta$ (defined as $d N / d \gamma \propto \gamma^{-\delta}$ ), a gas density jump by factor $x$ at the shock, and certain assumptions about the tangled magnetic field, the radio emissivity per unit volume would change as

$$
I_{\nu} \propto x^{\frac{2}{3} \delta+1} .
$$

If both compression and significant reacceleration are present, for a fixed observed post-shock radio brightness, we would expect a lower level of pre-shock radio emission, and in the case of the Fermi acceleration directly from the thermal pool, the pre-shock radio emission would be lower still by many orders of magnitude. With our new, higher-sensitivity radio map, we can try to test these possibilities by extracting a radio surface brightness profile across the shock.

\subsubsection{Modeling Radio Emissivity Profile}

In the same sector $\mathrm{N} 1+\mathrm{N} 2$ where we obtained the highest Mach number bins, we extracted a radio profile binned to the beam size and aligned with the best-fit shock position. It is shown in Figure 6(b); the radio brightness drops sharply at the position of the X-ray shock and is not detected in the pre-shock region. To evaluate measurement errors for the profile, we generated Gaussian noise images with the observed rms noise of $22 \mu \mathrm{Jy}$ beam $^{-1}$ after smoothing by the beam size, and extracted radial profiles from 1000 smoothed noise images. An 
elongated "hole" in the radio image in the $\mathrm{N} 1+\mathrm{N} 2$ sector about $200 \mathrm{kpc}$ in front of the shock (dashed radio contour in Figure 6(a)) is most likely an interferometric artifact. Since we want to place an upper limit on the pre-shock radio emission, to be conservative we masked this negative deviation.

We will now compare this radio brightness profile and, in particular, the non-detection in the pre-shock region, with the expectation for an adiabatic compression model for the origin of the radio edge. To model the radio image, we created a spherical model of the radio emissivity in the relevant region of space, projected it on the sky, and convolved it with the VLA beam.

For lack of information on the distribution of cosmic rays and magnetic fields in A520, our model makes two assumptions. First, the density of cosmic-ray electrons is assumed proportional to that of thermal ICM. If we consider the various possible sources for fossil electrons-merger shock acceleration and subsequent vigorous mixing, disrupted and mixed radio galaxies, turbulent acceleration, and "secondary" electrons from cosmic-ray proton collisions (see Brunetti \& Jones 2014 for a review) — this seems a reasonable assumption. We note that for our purpose, this is a conservative assumption compared to the alternative of a flat cosmic-ray density profile. Second, we assume the magnetic field strength changes across the cluster as $B \propto n^{0.5}$ (where $n$ is gas density), which is the best fit derived for Coma (Bonafede et al. 2010), also a merging cluster. Then the synchrotron emissivity (emission per unit volume) $P \propto n B^{2} \propto n^{2}$, so the pre-shock radio emissivity has essentially the same dependence on the gas density as the $\mathrm{X}$-ray. We therefore use the radial profile derived from the $\mathrm{X}$-ray and only let the normalization change to model the radio profile.

The post-shock region is fit very well with the projection of a 3D model with an abrupt emissivity drop, convolved with the beam. We assume constant emissivity versus radius in this region, because we are most interested in the bin immediately next to the shock surface and because it is not clear how the radio brightness should change further downstream (the image does not have enough leverage to fit this slope because of the unrelated radio structures inside the cluster halo). We did check that the post-shock radio profile does not favor, for example, a thin 3D shell that in projection would show a peak at the shock position and a decline toward smaller radii. The jump in radio emissivity at the X-ray shock location is the only free parameter. The model is truncated at $1.5 \mathrm{Mpc}$ from the X-ray centroid (which is $1.15 \mathrm{Mpc}$ from the shock surface); because of the model's steep decline, this does not matter much.

Because an interferometer can lose signal on large angular scales, we must be careful when deriving an upper limit in the low-surface-brightness areas. The unknown zero level of the image limits our ability to constrain the pre-shock emission, but we should be able to constrain models with steep changes on linear scales that are well within the nominal $u v$ coverage limit, which is $\sim 3 \mathrm{Mpc}$ for this data set (Section 3). However, there may be subtle artifacts on all scales, and for example, the apparent systematic negative values in the radio profile in the pre-shock region are a cause for concern. With this in mind, rather than simply fitting the projected radio emission model to the profile, we tried to account for the possible artifacts to a first approximation by convolving the brightness model with the actual $u v$ coverage and the beam and reconstructing the image. Technically, we followed Giacintucci et al. (2014) and "injected" or added our brightness model for the pre-shock emission into the pre-shock region of the data using the AIPS task UVSUB. We then extracted a radial profile in the same sector of the new image, thought of it as a model, and compared it with the actual profile using the $\chi^{2}$ statistics. (Because the same statistical noise is present in both the real and the "model" images, for the $\chi^{2}$ calculation we used the errors for only one of the profiles). The injected brightness model was calculated by keeping the post-shock emission at the same best-fit level, while varying the jump amplitude and thus the normalization of the pre-shock profile (only the preshock region of the model emission was injected). We compared the data and model profiles in the $400 \mathrm{kpc}$ pre-shock radial interval (Figure 6(b)) to avoid being affected by the accuracy of our model assumptions while being interested only in the shock jump.

This exercise revealed that the negative deviations in the preshock region are indeed an artifact-the difference between the image with and without the injection there was less than the injected emission, which means that the interferometer does redistribute the flux from this region into other radial bins (see, e.g., the positive bump around $x \approx 500 \mathrm{kpc}$ ). Theoretically, it should be possible to account for this effect and constrain the absolute brightness in the pre-shock region, but it would require creating an accurate spatial model of the radio brightness for the entire cluster, which is beyond the scope of this study. (It may be more efficient instead to obtain a data set with better $u v$ coverage that would not require such modeling.)

Nevertheless, we can evaluate the sensitivity of this radio image to the pre-shock emission under the assumption that the true pre-shock emission in the data is zero. Then, an injected model that corresponds to the emissivity jump by a factor of 10 (see Figure 6(b)) is rejected at a $3 \sigma$ statistical significance, while a jump by a factor of 16 is rejected at $2 \sigma$. If we ignored the interferometric artifact and simply convolved the brightness model with the beam without accounting for the $u v$ coverage, we would have excluded at a $3 \sigma$ level a jump by factor 22 .

\subsubsection{Comparison with Adiabatic Compression}

Let us now compare this with the emissivity jump expected in the adiabatic compression model. Compression should preserve the shape of the electron energy spectrum, while shifting it in frequency and changing its normalization. For a power-law electron spectrum, the radio synchrotron spectrum $I_{\nu} \propto \nu^{-\alpha}$ is related to the electron spectrum via $\alpha=(\delta-1) / 2$. For the radio spectral index, we use $\alpha=1.25 \pm 0.11$ ( $1 \sigma$ errors) from the first post-shock bin in Figure 6 of Vacca et al. (2014), which corresponds to $\delta=3.5 \pm 0.2$. Formally, for the observed gas density jump of $x=2.7$ in sector $\mathrm{N} 1+\mathrm{N} 2$ (Table 2), Equation (5) gives the expected radio emissivity jump of $27 \pm 4$ for the adiabatic compression scenario. For comparison with the data, we need to include projection effects, because the post-shock radial brightness profile includes regions along the los that are away from the shock "nose," with a lower density jump. The gas density jump azimuthal dependence in the plane of the sky in the sectors in which it was measured, can be interpolated well by $x=x_{\text {nose }}(\cos \theta)^{1 / 2}$, where $\theta$ is the angle from the "nose" of the shock. Then, we assumed rotational symmetry about the "nose" and calculated the shock-surface-area-weighted radio jump (given by Equation (5)) in the first post-shock bin in the radio brightness profile. This gives a value of 16 for the average radio emissivity 
jump, which can be directly compared with the limits derived above for a model that did not include this azimuthal dependence for simplicity.

If we approximately include radiative cooling of the postshock relativistic electrons, we expect a lower, more easily detectable emissivity jump at the shock. The radio spectrum should steepen within $\sim 100 \mathrm{kpc}$ downstream of the shock (M05). The beam size of the VLA data used to calculate spectral index images by Vacca et al. is $130 \mathrm{kpc}$ (see their Figure 4), so such a spectral change in the immediate postshock region is not resolved, and the spectral index immediately at the shock would be flatter. An unresolved mixture should have a volume-averaged slope of $\bar{\alpha} \approx \alpha+1 / 2$ (Ginzburg \& Syrovatskii 1964), so for $\bar{\alpha} \simeq 1.25$ the value at the shock could be $\alpha \simeq 0.75$, for which the radio emissivity jump in the compression scenario would be a factor of 9 (including the projection of oblique shock contributions), compared to 16 obtained above without cooling. The true value may be somewhere in this interval, depending on the processes in the post-shock plasma. Note that in the above calculations, we do not consider radiative cooling of the pre-shock electrons, in effect assuming either that something balances that cooling or that the pre-shock electrons are continuously generated by some process (e.g., cosmic-ray proton collisions with thermal protons). If cooling is balanced pre-shock, it may be balanced post-shock as well, so the above cooling correction for the spectral index would not be necessary. A high-resolution map of the post-shock spectral index may shed light on the relevant physical processes here.

Comparing these estimates to the limits above, we see that the statistical sensitivity of the radio data would allow us to exclude such jumps at $>3 \sigma$ confidence. However, because of the unfortunate interferometric artifact, the exclusion significance is lower, only $\sim 2 \sigma$, and it depends on the assumption about the zero level in the image. Nevertheless, this demonstrates that ruling out the compression model is within reach with a data set with similar sensitivity but better $u v$ coverage.

Note that our estimates above used the assumption that the electrons have a power-law energy spectrum. If this is not the case (e.g., both pre-shock and post-shock spectra may have a cutoff at some frequencies because of radiative cooling), the adiabatic model can still be constrained, but it requires measurements at several frequencies. As noted in M05, using our notation, a single electron emits most of its synchrotron radiation at a frequency that scales with the compression factor as

$$
\nu_{\text {peak }} \propto B \gamma^{2} \propto x^{4 / 3}
$$

For $x=2.7$, the post-shock electron emitting at $1.4 \mathrm{GHz}$ would have emitted at $370 \mathrm{MHz}$ before the shock passage (or at $560 \mathrm{MHz}$ for $x=2.0$ ). So pre-shock observations at those lower frequencies, combined with the post-shock $1.4 \mathrm{GHz}$ brightness, would be least dependent on the assumed shape of the electron spectrum. Alternatively, pre-shock measurements at $1.4 \mathrm{GHz}$ would need to be combined with higher-frequency data for the post-shock region. And, ultimately, measuring the spectrum of the post-shock emission in the relevant range above and below $1.4 \mathrm{GHz}$ and verifying that it is a power law (or detecting a curvature) would provide the most robust constraint.

\section{Summary}

We analyzed a deep Chandra exposure of A520 to study its prominent bow shock, one of only a handful of merger shocks with simple and unambiguous geometry and a relatively high Mach number. At the "nose" of the shock, we find $M=2.4_{-0.3}^{+0.4}$. This is higher than in the previous study based on a shorter exposure (M05), because we were able to use a narrower sector at the "nose" of the shock. As expected, the Mach number declines (toward 1.6-1.7) away from the "nose," where the shock front becomes oblique.

The relatively high Mach number of the central segment of the front allowed us to perform a test of the electron-proton equilibration timescale, similar to the earlier tests for the Bullet cluster (M06, MV) and A2146 (R12). We fit the shock X-ray brightness profile using a gas density model with a jump, used the density jump to evaluate the post-shock gasdynamic temperature, and compared it to the measured post-shock electron temperature. The electron temperature immediately behind the shock is higher than expected from a simple picture where electrons are compressed adiabatically by the shock and then equilibrate with protons on a Coulomb collisional timescale. This indicates a faster equilibration rate, pointing to the prevalence of other particle interactions in hot magnetized plasma. Although the confidence level is only 95\% (this includes the statistical error on temperature, ACIS background uncertainty, and sky background effect), it is similar to the finding for the Bullet cluster (M06; MV). Although the A2146 result (R12) was inconclusive (mostly because its Mach number is lower and the amplitude of the effect is smaller), it did prefer adiabatic compression over fast equilibration. The scatter between these results most likely reflects the geometric uncertainty inherent in this test-the curvature of the shock front in the sky plane is used to model its curvature along the los. This scatter can be averaged out by studying a sample of shocks, and our result provides a third entry for such a sample. Unfortunately, bow shocks that are as clear cut as Bullet or A520 are rare, so expanding the sample may require going to higher redshifts with more sensitive instruments. This is worth the effort, because cluster shocks provide one of the most direct methods of determining this important timescale for any astrophysical plasmas.

We also present a new combined analysis of the archival $1.4 \mathrm{GHz}$ radio VLA data on the cluster giant radio halo, previously analyzed separately in Govoni et al. (2001) and Vacca et al. (2014). In addition to providing lower statistical noise, the data sets complement each other's interferometric coverage, which improves fidelity of the reconstructed image. The radio image reveals several interesting features, such as the bright spot that coincides with the disrupted cool core, possibly related to a former minihalo. Another bright spot may point to a region of high turbulence, a possible target for future X-ray calorimetric measurements.

A520 is one of the growing number of clusters where both a giant radio halo and an X-ray shock front are observed (Markevitch 2012). As in most of them, there is a prominent sharp edge of the radio halo that coincides with the X-ray shock front. Some clusters have X-ray shocks with counterparts both in the form of the halo edges and radio relics (Shimwell et al. 2015). Studying these colocated features may shed light on the physical processes responsible for the generation and acceleration of the radio-emitting electrons. For example, in our A520 data set, the radio emission in the pre-shock region is 
undetected at a very low brightness level, which has not been probed for any other shocks. If the jump of the radio emission at the shock were caused by simple adiabatic compression of relativistic electrons in the pre-shock plasma (e.g., remaining from past shocks or produced throughout the cluster by cosmicray proton interactions), we should see the radio emission beyond the edge (M05). We came close to being able to rule this model out (and thus demonstrate the existence of particle acceleration or reacceleration at shocks) based on statistical sensitivity of the radio data. However, an interferometric artifact in the region of interest dominates the uncertainty. Our analysis shows that this interesting test for the cluster radio halos is within reach, but probably requires an observation with a better interferometric coverage and at lower frequencies, e.g., with GMRT or LOFAR.

We thank the anonymous referee for valuable criticisms that led us to more accurate conclusions. Q.H.S.W. was supported by Chandra grants GO3-14144Z, GO5-16147Z, and AR516013X. S.G. acknowledges the support by the National Aeronautics and Space Administration, through Chandra Award Numbers AR5-16013X and G05-16136X. Basic research in radio astronomy at the Naval Research Laboratory is supported by 6.1 Base funding.

\section{ORCID iDs}

Qian H. S. Wang (1) https://orcid.org/0000-0001-8468-9164

Simona Giacintucci 주 https://orcid.org/0000-0002-1634-9886

Maxim Markevitch (1) https://orcid.org/0000-0003-0144-4052

\section{References}

Anders, E., \& Grevesse, N. 1989, GeCoA, 53, 197

Blandford, R., \& Eichler, D. 1987, PhR, 154, 1
Bonafede, A., Feretti, L., Murgia, M., et al. 2010, A\&A, 513, A30

Brunetti, G., \& Jones, T. W. 2014, IJMPD, 23, 30007

Cassano, R., Ettori, S., Brunetti, G., et al. 2013, ApJ, 777, 141

Clarke, T. E., \& Ensslin, T. A. 2006, AJ, 131, 2900

Feretti, L., Giovannini, G., Govoni, F., \& Murgia, M. 2012, A\&ARv, 20, 54

Fox, D. C., \& Loeb, A. 1997, ApJ, 491, 459

Giacintucci, S., Markevitch, M., Brunetti, G., et al. 2014, ApJ, 795, 73

Giacintucci, S., Markevitch, M., Cassano, R., et al. 2017, ApJ, 841, 71

Giacintucci, S., Venturi, T., Macario, G., et al. 2008, A\&A, 486, 347

Ginzburg, V. L., \& Syrovatskii, S. I. 1964, The Origin of Cosmic Rays (New York: Macmillan)

Govoni, F., Feretti, L., Giovannini, G., et al. 2001, A\&A, 376, 803

Landau, L. D., \& Lifshitz, E. M. 1959, Fluid Mechanics (Oxford: Pergamon Press)

Markevitch, M. 2006, in ESA Special Publication 604, The X-ray Universe, ed. A. Wilson (Noordwijk: ESA), 723

Markevitch, M. 2012, arXiv:1010.3660

Markevitch, M., Govoni, F., Brunetti, G., \& Jerius, D. 2005, ApJ, 627, 733

Markevitch, M., Mushotzky, R., Inoue, H., et al. 1996, ApJ, 456, 437

Markevitch, M., \& Vikhlinin, A. 2007, PhR, 443, 1

Mazzotta, P., Rasia, E., Moscardini, L., \& Tormen, G. 2004, MNRAS, 354, 10 Perley, R. A., \& Butler, B. J. 2013, ApJS, 204, 19

Planck Collaboration, Ade, P. A. R., \& Aghanim, N. 2013, A\&A, 554, A140

Rees, M. J., Begelman, M. C., Blandford, R. D., \& Phinney, E. S. 1982, Natur, 295, 17

Russell, H. R., McNamara, B. R., Sanders, J. S., et al. 2012, MNRAS, 423, 236

Sarazin, C. L. 1988, X-ray Emission from Clusters of Galaxies (Cambridge: Cambridge Univ. Press)

Sarazin, C. L., Finoguenov, A., Wik, D. R., \& Clarke, T. E. 2016, ApJ, submitted (arXiv:1606.07433)

Shimwell, T. W., Brown, S., Feain, I. J., et al. 2014, MNRAS, 440, 2901

Shimwell, T. W., Markevitch, M., Brown, S., et al. 2015, MNRAS, 449, 1486

Spitzer, L. 1962, Physics of Fully Ionized Gases (2nd ed.; New York: Interscience)

Takizawa, M. 1999, ApJ, 520, 514

Vacca, V., Feretti, L., Giovannini, G., et al. 2014, A\&A, 561, A52

van Weeren, R. J., Röttgering, H. J. A., Brüggen, M., \& Hoeft, M. 2010, Sci, 330,347

Wang, Q. H. S., Markevitch, M., \& Giacintucci, S. 2016, ApJ, 833, 99

Wong, K.-W., \& Sarazin, C. L. 2009, ApJ, 707, 1141

Zeldovich, Y. B., \& Raizer, Y. P. 1966, Elements of Gasdynamics and the Classical Theory of Shock Waves (New York: Academic Press) 